\title{
High efficiency X-ray nanofocusing by the blazed stacking of binary zone plates
}

\author{
I. Mohacsia ${ }^{\mathrm{a}}$ P. Karvinen ${ }^{\mathrm{a}}$, I. Vartiainen ${ }^{\mathrm{a}}$, A. Diaz ${ }^{\mathrm{a}}$, A. Somogyi ${ }^{\mathrm{b}}$, C. M. Kewish ${ }^{\mathrm{b}}$, P. Mercere ${ }^{\mathrm{b}}$, \\ C. David ${ }^{\mathrm{a}}$ \\ ${ }^{a}$ Paul Scherrer Institut, 5232 Villigen, Switzerland \\ ${ }^{\mathrm{b}}$ Synchrotron SOLEIL, L'Orme des Merisiers Saint-Aubin 91192 Gif-sur-Yvette, France
}

\begin{abstract}
The focusing efficiency of binary Fresnel zone plate lenses is fundamentally limited and higher efficiency requires a multi step lens profile. To overcome the manufacturing problems of high resolution and high efficiency multistep zone plates, we investigate the concept of stacking two different binary zone plates in each other's optical near-field. We use a coarse zone plate with $\pi$ phase shift and a double density fine zone plate with $\pi / 2$ phase shift to produce an effective 4step profile. Using a compact experimental setup with piezo actuators for alignment, we demonstrated $47.1 \%$ focusing efficiency at $6.5 \mathrm{keV}$ using a pair of $500 \mu \mathrm{m}$ diameter and $200 \mathrm{~nm}$ smallest zone width. Furthermore, we present a spatially resolved characterization method using multiple diffraction orders to identify manufacturing errors, alignment errors and pattern distortions and their effect on diffraction efficiency.
\end{abstract}

Keywords: Fresnel zone plate, $x$-ray nanofocusing, x-ray microscopy

\section{INTRODUCTION}

Various X-ray microscopy techniques are available for the user community, using either direct imaging or scanning based methods [1]. Obtaining high spatial resolution and photon flux to user experiments makes focusing optics key element of X-ray microscopy setups. Binary Fresnel zone plate lenses are often used as compact, flexible and easy to align focusing elements, but they have two fundamental disadvantages when compared to Kirkpatrick-Baez mirrors. First, diffractive optics are chromatic, making it difficult to use them in spectroscopy applications, where the variation of the incoming photon energy changes the focal distance. Second, they have equally strong positive and negative diffraction orders, meaning that half of the diffracted intensity is scattered into the divergent negative orders. The focusing efficiency of the $+1^{\text {st }}$ diffraction order is fundamentally limited up to $40.5 \%$ in case of non-absorbing structures with the optimal $\pi$ phase shift. As the phase shift is directly proportional to structure height and the resolution of Fresnel zone plates is connected to the smallest zone width, the fabrication of high resolution and high efficiency Fresnel zone plates requires the fabrication of high aspect ratio nanostructures especially in the multi-keV range.

To ease the compromise between resolution and efficiency Maser et al. [2] developed the concept of zone plate stacking. Two identical zone plates stacked in each others near field act as a single zone plate with doubled zone height. As the resultant zone plate stack is still a binary zone plate, it is still subjected to the fundamental efficiency limit. While a symmetric binary structure has equal positive and negative orders, asymmetric multistep profiles can have enhanced positive (focusing) orders due to the suppression of the negative (divergent) orders. This effect is generally known as blazing and can be used to reach diffraction efficiencies beyond the limit of a binary structure. In case of a four step profile diffraction efficiencies up to $81.1 \%$ become possible [3]. The fabrication of high resolution multistep zone plates is extremely difficult for small zone widths due to the resulting high aspect ratios and the required alignment accuracy between subsequent processing steps. Therefore they have so far only been demonstrated for rather coarse zone structures using $1 \mu \mathrm{m}$ smallest zone width [4].

The concept of multistep zone plates and zone plate stacking was combined by Chen et al.[5] by stacking two different binary zone plates, a "coarse" zone plate with $\pi$ phase shift and a double density "fine" zone plate with $\pi / 2$ phase shift in each others near field to obtain an effective four step profile (see Figure 1). However the resulting zone plates only achieved $29 \%$ diffraction efficiency, which could be achieved by binary zone plates.

In this paper we investigate a similar concept by extending it with a new kind of spatially resolved efficiency measurement to identify manufacturing and alignment related errors. This allowed us to achieve focusing efficiencies up to $47.1 \%$, while maintaining high spatial resolution with $200 \mathrm{~nm}$ smallest zone width. 


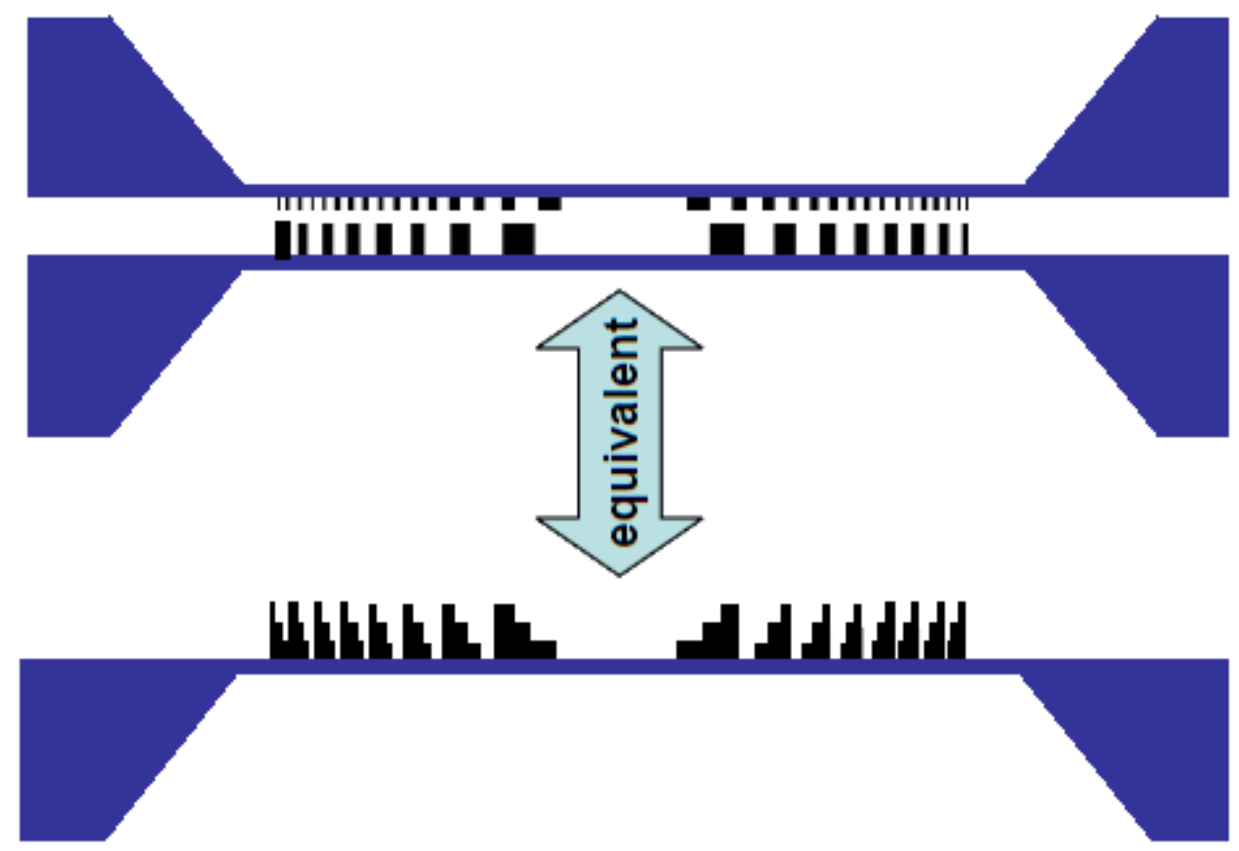

Figure 1: The stacking of two binary zone plates in each others near field with $\pi$ and $\pi / 2$ phase shift to produce an effective 4 step profile.

\section{SAMPLE FABRICATION}

We used the fabrication method developed by Gorelick et al. [6] for producing high aspect-ratio metallic nanostructures using the direct e-beam writing of PMMA resist mold. The zone plates were designed as large aperture diffractive focusing optics with $500 \mu \mathrm{m}$ diameter and $200 \mathrm{~nm}$ smallest zone width for the coarse and $100 \mathrm{~nm}$ smallest zone width for the dense zone plates. The lenses were designed to provide optimal performance at $6.5 \mathrm{keV}$, therefore the lenses were made of $2.4 \mu \mathrm{m}$ and $1.2 \mu \mathrm{m}$ high nickel structures corresponding to the required $\pi$ and $\pi / 2$ phase shift. At this energy, binary nickel zone plates are limited to $37 \%$ efficiency due to absorption losses, but four step profiles can go up to $71 \%$ [3]. The focal distance of the stack was determined by the coarse zone plate to be $524 \mathrm{~mm}$ at the selected energy.

The zone plates were prepared on $250 \mathrm{~nm}$ thick $\mathrm{Si}_{3} \mathrm{~N}_{4}$ membranes on $\mathrm{Si}$ support frames. The membranes were coated with a plating base and spin-coated with PMMA resist. The zone plate patterns were exposed into the resist layer by a $100 \mathrm{keV}$ Vistec EBPG 5000plus electron beam writer. The pattern diameter was small enough to fit into a single $500 \times 500 \mu \mathrm{m}^{2}$ write field, therefore no write field stitching was required. The development was performed by dipdevelopment in a mixture of isopropanol and water (7:3 by volume) [7]. The PMMA mold was filled with nickel electroplating using pulse-plating to obtain uniform structure height [8].

The obtained structures were characterized using optical and scanning electron microscopy (SEM), see Figure 2, and tested at synchrotron beamlines to characterize their behavior as individual and stacked elements. 


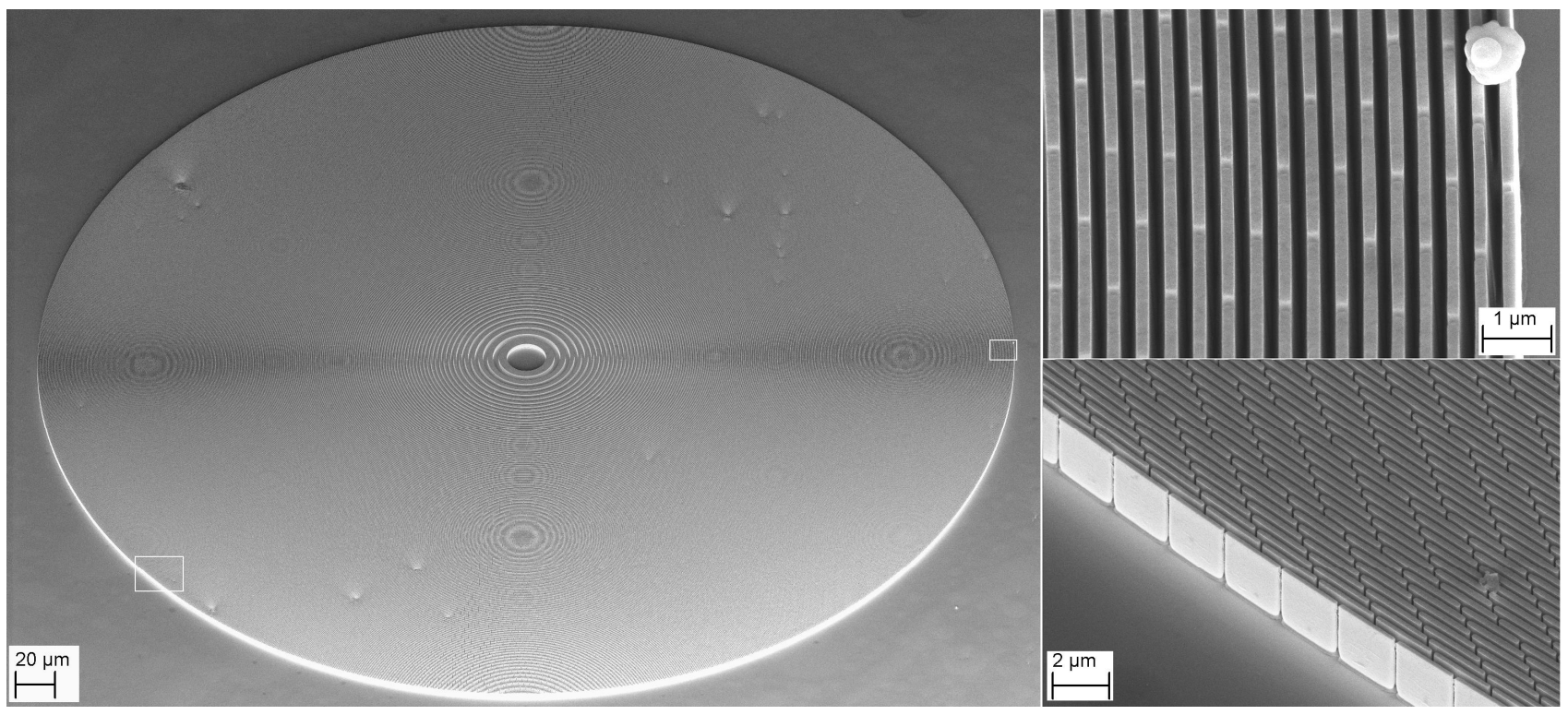

Figure 2: Scanning electron microscope images of a coarse Nickel zone plate. Left: Overview of a $500 \mu \mathrm{m}$ diameter coarse zone plate. Right: Outer zone plate regions showing zones of $200 \mathrm{~nm}$ line width and $2.4 \mu \mathrm{m}$ structure height.

\section{EXPERIMENTAL METHODS AND MEASUREMENTS}

The selected zoneplates were characterized at the cSAXS beamline of SLS, later final diffraction efficiency measurements were performed on the updated zone plates at the Metrology beamline of the Synchrotron SOLEIL. All measurements were performed on zone plates with $500 \mu \mathrm{m}$ diameter and $200 \mathrm{~nm}$ smallest zone width and at the photon energy of $6.5 \mathrm{keV}$.

\section{Integral focusing efficiency measurements of individual and stacked zone plates}

We measured the focusing efficiency of individual and stacked Fresnel zone plates by illuminating the zone plate through an upstream reference pinhole of equal diameter and selecting the diffraction order by placing a $20 \mu \mathrm{m}$ pinhole as order selecting aperture (OSA) in the selected order's focal plane. A photodiode detector was used to measure the intensity of the beam downstream of the focus. The pinhole was scanned across the focal plane, recording the background while the focal spot is outside and a sharp plateau when the focal spot is within the OSA. The background was subtracted from the plateau's value and the measured flux was converted to efficiency by normalizing to the intensity passing through the reference pinhole with no zone plate or OSA in the beam path.

To select the best candidates for stacking experiments, we performed focusing efficiency measurements on both the coarse and the dense zone plates individually. A coarse zone plate with $23.8 \%$ focusing efficiency was chosen, as the most efficient candidate. The dense zone plate was selected by having the lowest ratio of the $+1^{\text {st }}$ and $+2^{\text {nd }}$ diffraction orders, as this proved to be a good indicator for a close match of the zone width to its optimum value. The selected zone plates were stacked in each others near field using a compact piezo actuator based experimental setup, which allowed the high precision remote-controlled positioning of the lenses. Optical microscopy was used to pre-align the stack of zone plates to be within the range of the fine alignment piezo. The alignment was then refined using X-rays, by scanning the fine alignment piezo actuators while maximizing the flux through the OSA.

The measured $36.8 \%$ focusing efficiency was unexpectedly far below both the expected and the theoretical values. Therefore we investigated the cause of the unexpectedly low diffraction efficiency using a different experimental setup, as described in the following section. 


\section{Spatially resolved efficiency measurements of stacked zone plates}

To determine the cause of the low efficiency and to optimize the measurement time, we replaced the photodiode detector with a $7 \mathrm{~m}$ - long flight tube, followed by a Pilatus $2 \mathrm{M}$ photon counting pixel detector. We recorded the spatial variation of the intensity of the diverged (de-focused) $+1^{\text {st }}$ diffraction order. After normalizing with the background illumination, the resulting spatial efficiency map shows a highly inhomogeneous efficiency map (Figure 4a). Certain areas around the edge of the zone plate stack have negligible contribution to the total diffracted intensity, giving us the averaged efficiency of $36.8 \%$.

To characterize the dark areas, we had to identify, to where the incoming intensity was diffracted. A detailed wavefront characterization method for X-ray optics was demonstrated by Vila et al. [9] to characterize the wave front of Fresnel zone plates around the focal spot. However the method requires the coherent illumination of the zone plate and the resolution of the reconstructed wavefront has insufficient resolution in the zone plate plane to identify error sources. High resolution reconstruction of the zone plate would provide an alternative, but the acquisition time would be unfeasibly long, on the order of several days, due to the large area of the zone plate.

Therefore we developed a high throughput method by mounting a $30 \mu \mathrm{m}$ diameter upstream pinhole alongside the reference pinhole and removing the order selecting aperture. We record the diffraction pattern of a small area of the stack, by illuminating it through a $30 \mu \mathrm{m}$ upstream pinhole (Figure 3). The recorded diffraction pattern shows discrete diffraction peaks, including both positive and negative orders, as locally the zone plate is similar to gratings. By scanning the upstream pinhole, we obtained a spatially resolved intensity map of the diffraction orders. The map reveals that the ratio of the $+1^{\text {st }}$ and $-1^{\text {st }}$ diffraction orders change significantly across the zone plate area, with the $+1^{\text {st }}$ order decreasing and the $-1^{\text {st }}$ increasing in the areas corresponding to the dark areas in the diffraction cone (Figure $4 \mathrm{~b}$ ).

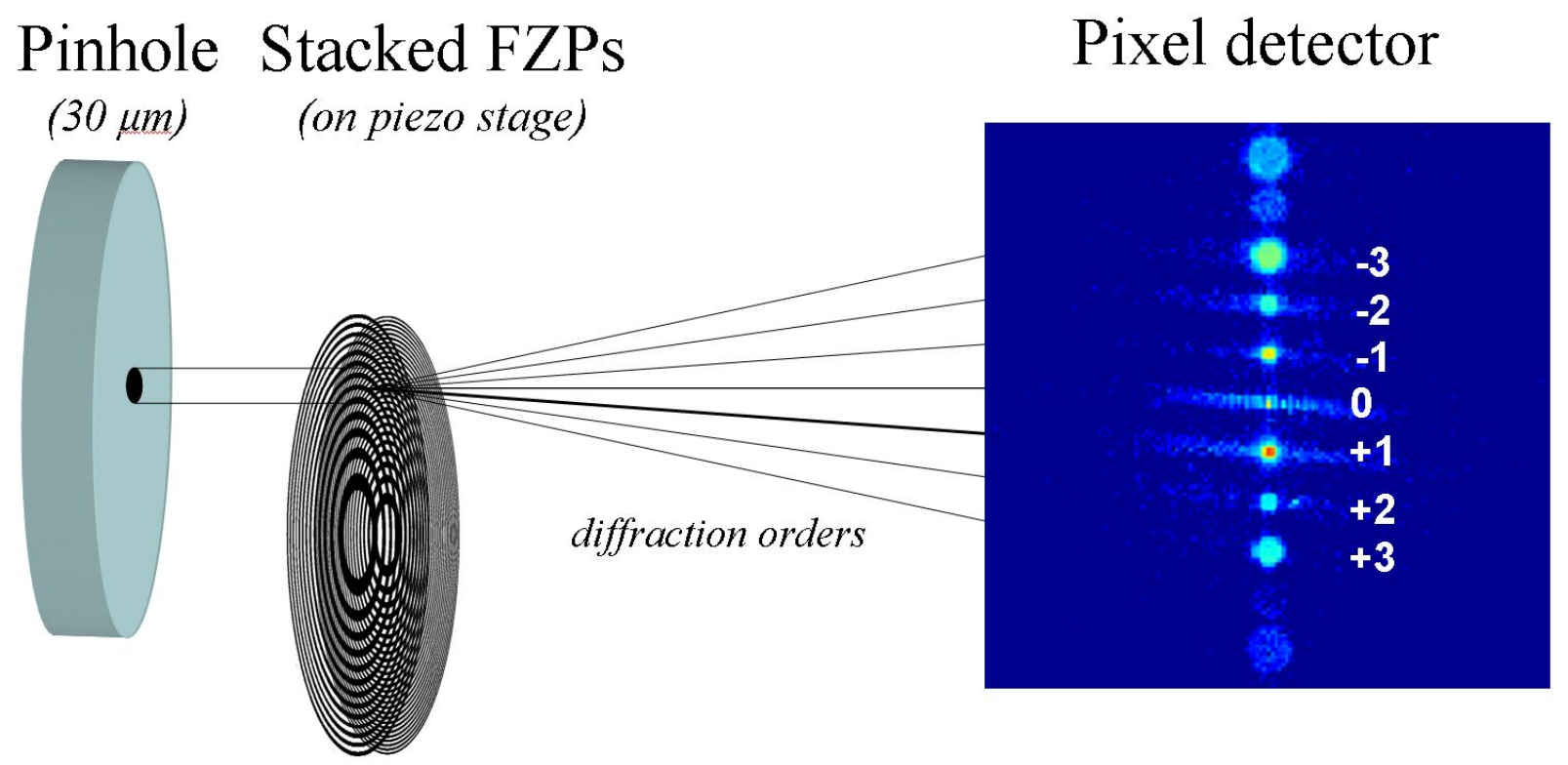

Figure 3: Experimental setup for spatially resolved efficiency measurements on Fresnel zone plates. As the zone plate is locally similar to a grating, we observe discrete diffraction peaks. Note that the focusing +1 st diffraction order is much stronger than the divergent -1st order, showing the concept of multistep zone plates. 

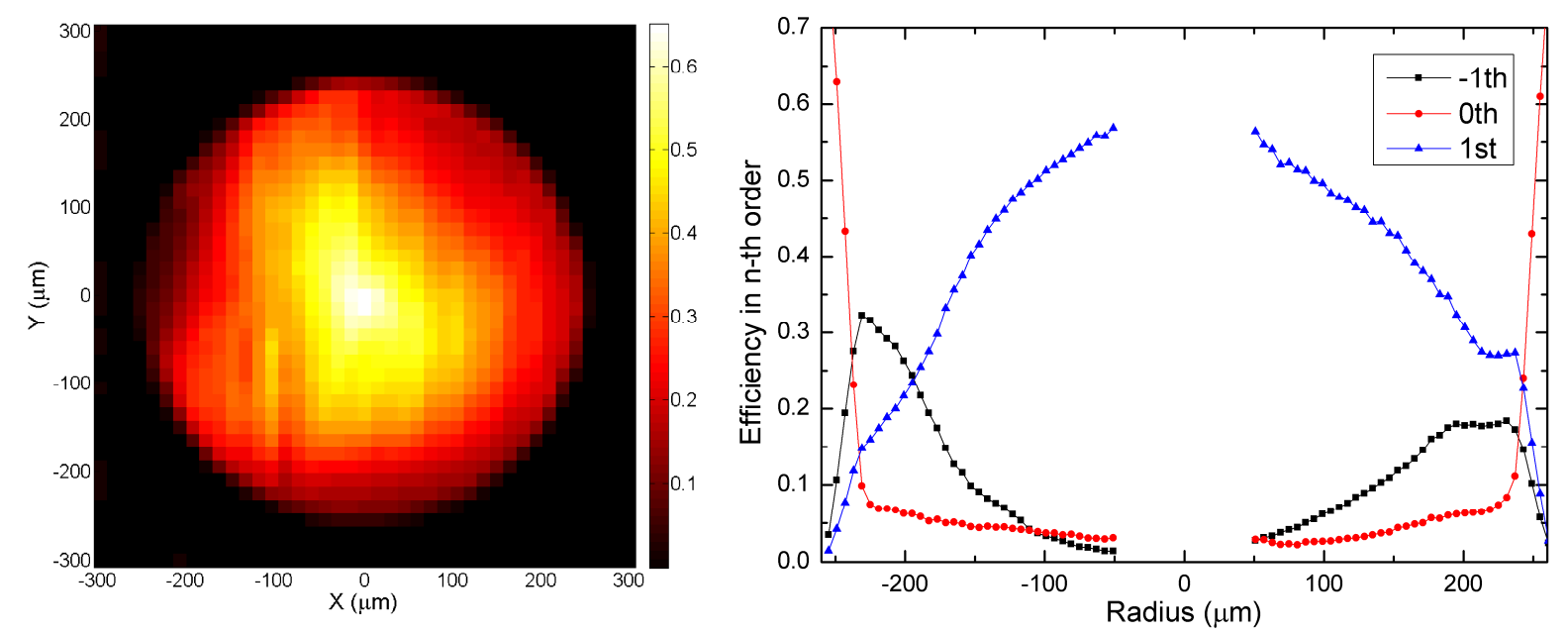

Figure 4: Spatial efficiency distribution diffraction cone (left). The large central structures are tolerant to errors, having focusing efficiency close to the theoretical value, but the efficiency drops quickly in the outer regions of smaller zone width. Distribution of major diffraction orders across the diagonal, obtained by scanning the upstream pinhole (right). This shows that the drop in the $1^{\text {st }}$ order efficiency is followed by an increase in the $-1^{\text {st }}$ order. The range, where the $-1^{\text {st }}$ order is stronger than the $+1^{\text {st }}$ indicates strong misalignment. It corresponds to anti-blazing: i.e. a staircase oriented in the opposite direction.

This is demonstrated on Figure 4, where the large sized central zones show the expected blazing scheme with increased focusing efficiency in the $+1^{\text {st }}$ order and suppressed $-1^{\text {st }}$ order. However the efficiency in the $+1^{\text {st }}$ order decreases towards the edges of the stack and the $-1^{\text {st }}$ order increases up to a point where it becomes even stronger than the $+1^{\text {st }}$ order. This corresponds to "anti-blazing" where the $-1^{\text {st }}$ order is enhanced and the $+1^{\text {st }}$ suppressed, meaning that the staircase is oriented in the opposite direction than intended. This implies that while the stack is in the globally best alignment, some areas of the stack are locally misaligned by more than $1 / 4^{\text {th }}$ of local zone width. This can be explained by relative distortions of the two zone plates preventing the perfect overlap of the full zone plate area. The dark areas can be perfectly aligned when shifted by the local misalignment, as demonstrated in Figure 5. The figure also shows that the intensity of the $+1^{\text {st }}$ and $-1^{\text {st }}$ orders changes in counter phase with the misalignment. Since the stacking implemented the asymmetry to the system, their ratio only depends on the local misalignment of the two zone plates.
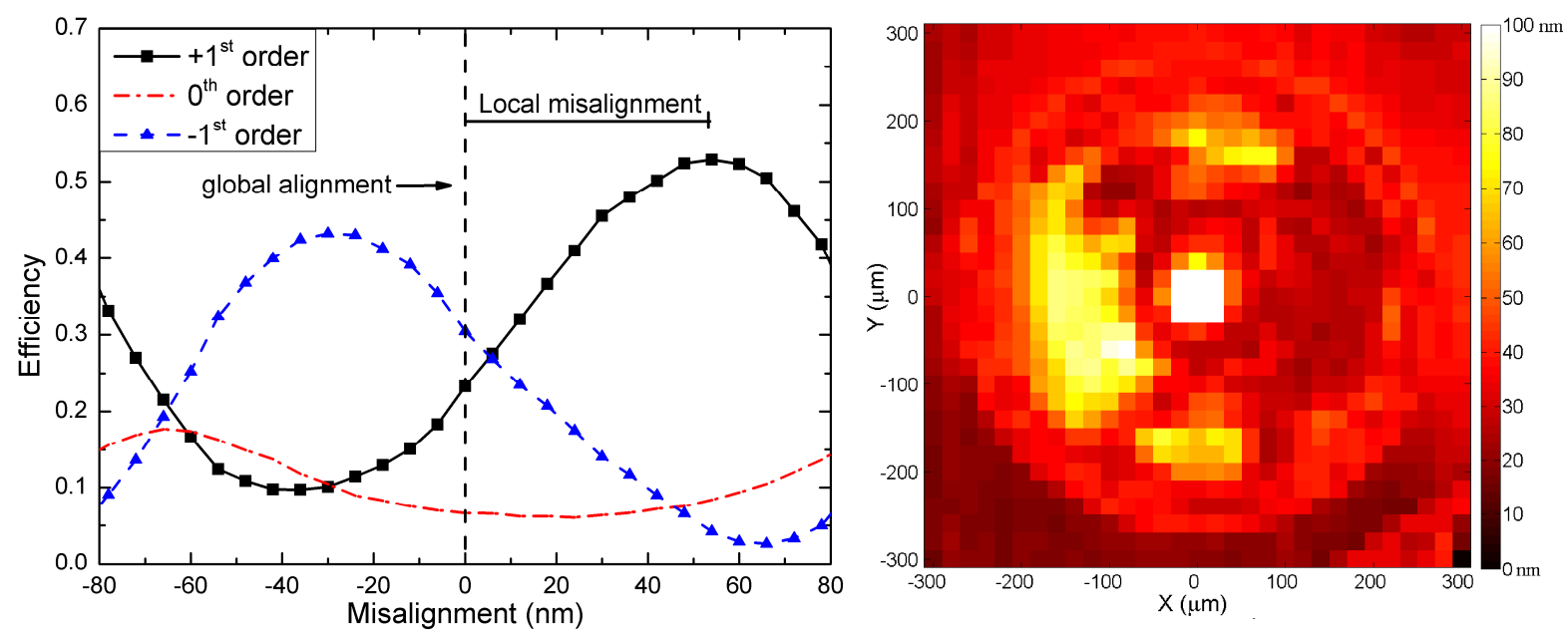

Figure 5: Scanning the alignment piezo, while looking at a fixed area of the stack (left). This demonstrates that the local alignment does not coincide to the global alignment of the stack. This indicates that pattern distortions prevent the alignment of the whole zone plate area. The efficiency of the $+1^{\text {st }}$ and $-1^{\text {st }}$ orders move in counter phase with the alignment. Therefore the spatial map of the $+1^{\text {st }}$ and $-1^{\text {st }}$ orders can be converted into the spatial map of relative distortions (right). This reveals that relative distortions up to $100 \mathrm{~nm}$ are present between the stacked binary zone plates. 
This allows the conversion of the measured intensities to local misalignment with sub-10 $\mathrm{nm}$ precision. The actual spatial mapping used a coarse sampling throughout the zone plate area, resulting in a significant speedup in acquisition time, when compared to direct high resolution measurements. However, the important parameters could be obtained with much higher precision. The intensity of the different diffraction orders is connected to the cross section of the zones via the Fourier transformation. Using this relationship reversely, we measure the Fourier series of the cross section of the zones. The method is not limited to the characterization of stacked zone plates, but can be applied to most binary and multilevel diffractive X-ray optics.

The map reveals that relative distortions up to $100 \mathrm{~nm}$ are present between the two zone plates in the stack. The distortions are concentrated in discrete areas roughly corresponding to the dark areas in the diffraction cone.

Direct mapping of distortions was performed on a Leica LION LV-1 electron beam writer in SEM mode using the interferometric stage for precise sample movement. We tracked the position of a zone close to the zone plate edge around the circumference and compared its radius with the predicted values. The metrological mapping revealed distortions up to $\pm 70 \mathrm{~nm}$, the major component of the distortions appears to be in phase in both zone plates, resulting in an ellipsoidal distortion. The orientation of the distortions matches the diagonal of the e-beam writer's write field, and can be attributed to tilted substrate or not exactly perpendicular axes of the e-beam writer. The local misalignment due to the ellipsoidal component can be significantly reduced with proper rotational alignment during the stacking procedure, namely by rotating the two zone plates by 90 degrees in respect to the electron beam writers write field, thus bringing the two ellipses in overlap.
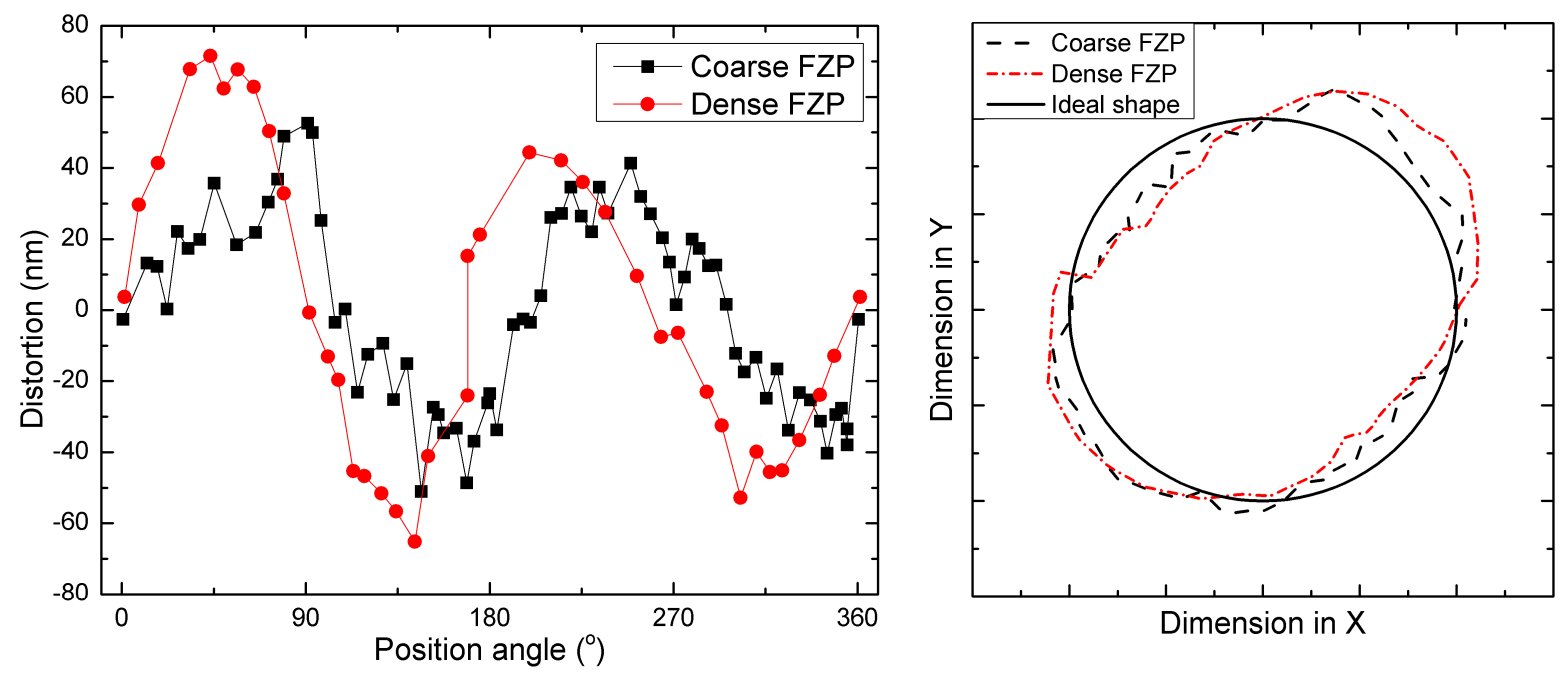

Figure 6: Deviation of the radius of two zone plates measured around the zone plate circumference close to the zone plate edges using a metrology SEM (left). Similar distortions can be observed over both the coarse and the fine zone plate. The elliptic shape of the distortions is enhanced for visualization on the right picture; note that the distortions are not exactly elliptical. The real distortions are in the order of $1 / 5000$. 

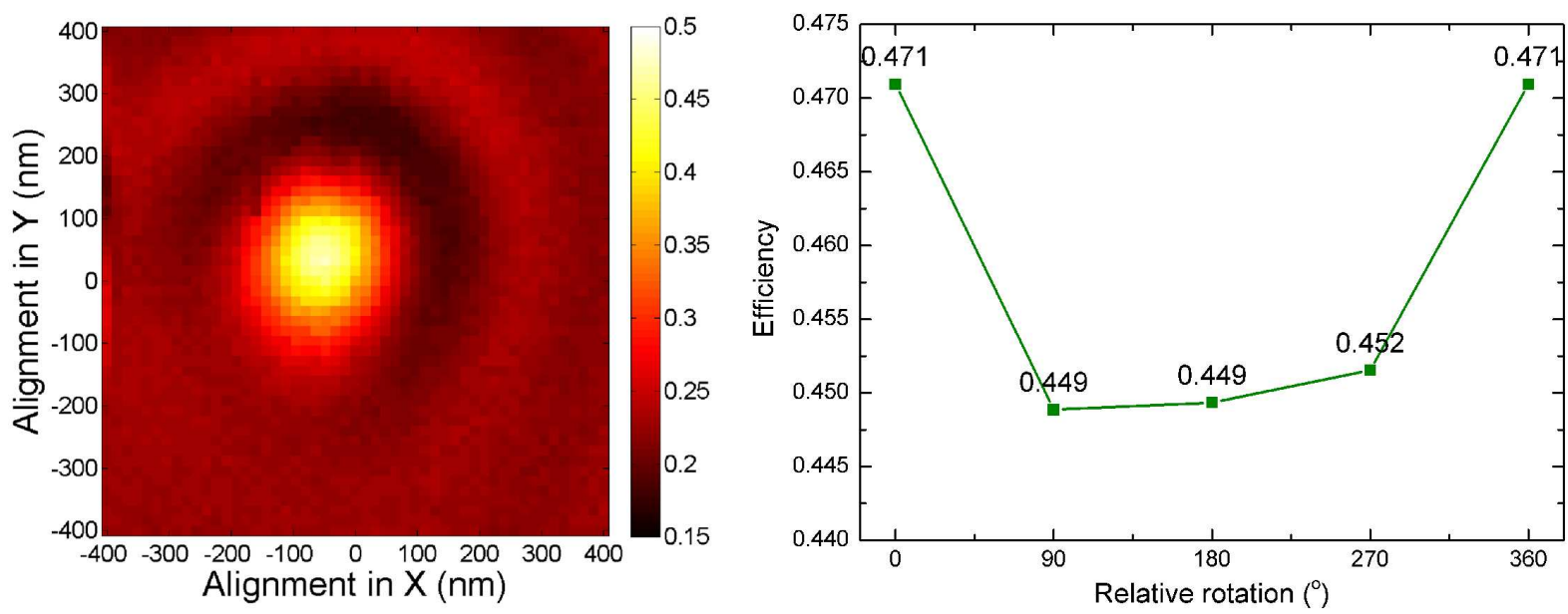

Figure 7: Diffraction efficiency of optimized zone plates as a function of alignment around the common optical axis, measured at $0^{\circ}$ orientation and peaking at $47.1 \%$ (left). The alignment is tolerant to small misalignment around the optical axis. Peak efficiency of stacked zone plates as a function of relative rotational orientation (left). The focusing efficiency shows a definite peak at $0^{\circ}$, but the small amplitude of the variations suggests that rotation only had a minor effect on focusing efficiency (right).

\section{Final efficiency measurements on individual and stacked zone plates}

The final diffraction efficiency measurements were performed on a new version of zone plates. We optimized exposure parameters to minimize line broadening towards the bottom of the structures. Special care was taken to minimize substrate tilt during the exposure. The individual coarse zone plate selected for stacking had $31.0 \%$ diffraction efficiency. The selected zone plate was stacked with a suitable dense zone plate based on prior SEM inspections. Diffraction efficiency was measured in multiple orientations with the zero point being the supposed optimal alignment.

The diffraction efficiency of stacked zone plates was measured by a high resolution scan with the fine alignment piezo, covering the whole alignment peak around the common optical axis (Figure 7). This method was chosen to ensure that the zone plates were measured in the best alignment and also provided information about the stack's behavior to small misalignment. The measurement resulted in measured diffraction efficiencies up to $47.1 \%$, with the best efficiency being at the expected rotational orientation (Figure 7). The measurement did not indicate large variations due to rotation only a $+4 \%$ variation. Therefore the increase in focusing efficiency can be attributed to the combined effect of improved individual lens quality, minimized distortions due to substrate tilt and rotational alignment.

\section{CONCLUSIONS}

In this paper we have demonstrated the feasibility of surpassing the fundamental efficiency limit of binary Fresnel zone plates in the hard X-ray regime by stacking two binary Fresnel zone plates into an effective staircase profile. We systematically identified sources of error behind our previous experiments using an acquisition time optimized method. The identified issues were solved using a combination of improved lens quality, minimizing distortions and applying rotational alignment. In our setup we have demonstrated $47.1 \%$ focusing efficiency at $6.5 \mathrm{keV}$ in the focused $+1^{\text {st }}$ diffraction order over a diameter of $500 \mu \mathrm{m}$, while maintaining high resolution and reasonable focal distance with 200 $\mathrm{nm}$ smallest zone width. When illuminated with coherent X-ray beam, we expect a spot size of about $250 \mathrm{~nm}$. From this we can estimate the gain of the zone plates to be beyond $10^{6}$.

\section{ACKNOWLEDGEMENTS}

Initial Fresnel zone plate lens characterization measurements were performed on the cSAXS beamline (X12SA) at the Swiss Light Source, Paul Scherrer Institut (Villigen, Switzerland). The authors acknowledge synchrotron SOLEIL (Gifsur-Yvette, France) for provision of synchrotron radiation facilities at the Métrologie beamline for the diffraction efficiency characterization measurements. 


\section{REFERENCES}

[1] Kaulich, B., Thibault, P., Gianoncelli, A., Kiskinova, M., "Transmission and emission x-ray microscopy: operation modes, contrast mechanisms and applications" J. Phys. Condens. Matter 23, 083002 (2011).

[2] Maser, J., Lai, B. P., Yun, W., Shastri, S. D., Cai, Z., Rodrigues, W., Xu, S., Trackhtenberg, E., "Near-field stacking of zone plates for hard x-ray range" Proc. SPIE 4783, 74 ( 2002).

[3] Kirz, J., "Phase zone plates for x rays and the extreme uv" JOSA, 64, 301 (1974).

[4] Di Fabrizio, E., Romanato, F., Gentili, M., Cabrini, S., Kaulich, B., Susini, J., Barrett, R., "High-efficiency multilevel zone plates for keV X-rays" Nature 401, 895-898 (1999).

[5] Chen, S., Lyon, A., Kirz, J., Seshadri S.; Feng J.; Feser M.; Sassolini S.; Duewer F.; Zeng X.; Huang C., "Absolute efficiency measurement of high-performance zone plates" Proc. SPIE 7448, 9 (2009).

[6] Gorelick S., Guzenko V. A., Vila-Comamala J., David C., "Direct e-beam writing of dense and high aspect ratio nanostructures in thick layers of PMMA for electroplating" Nanotechnology 21, 295303 (2010).

[7] Shazia, Y., Hasko, D. G., Ahmed H., "Comparison of MIBK/IPA and water/IPA as PMMA developers for electron beam nanolithography" Microelectronic Engineering 61, 745 (2002).

[8] Lindblom, M., Hertz, H. M., Holmberg, A., "Pulse reverse plating for uniform nickel height in zone plates" J. Vac. Sci. Technol. B 24, 2848 (2006).

[9] Vila-Comamala, J., Diaz, A., Guizar-Sicairos, M., Mantion, A., Kewish, C. M., Menzel, A., Bunk, O., David, C., "Characterization of high-resolution diffractive X-ray optics by ptychographic coherent diffractive imaging" Optics Express, 19, 22, 21333-21344 (2011). 